\title{
An Implementation of a Texture Analysis System
}

\author{
Ronald Pandolfi \\ Office of Science, Science Undergraduate Laboratory Internship \\ Program
}

University of California, Merced

Stanford Synchrotron Radiation Lightsource
Stanford, California

August 6, 2009

Prepared in partial fulfillment of the requirement of the Office of Science, Department of Energy's Science Undergraduate Laboratory Internship under the direction of Apurva Mehta in Stanford Synchrotron Radiaion Lightsource division of the Stanford Linear Accelerator Center.

Participant:

Signature

Research Advisor:

Signature 


\section{Contents}

1 Introduction $\quad 4$

2 Diffraction Geometry $\quad 5$

3 Methods $\quad 5$

4 Conclusions $r$

5 Acknowledgements $\quad 9$ 


\begin{abstract}
An Implementation of a Texture Analysis System. RONALD PANDOLFI (University of California, Merced 95243) APURVA MEHTA (Stanford Synchrotron Radiation Lightsource, Stanford, CA 94025) MATT BIBEE (Stanford Synchrotron Radiation Lightsource, Stanford, CA 94025) JAMES (RANDY) GROVES (Stanford Synchrotron Radiation Lightsource, Stanford, CA 94025).
\end{abstract}

From analysis of the crystalline texture (the distribution of crystalline orientations in a polycrystalline substance) of target samples, some critical material properties of those samples may be deduced. Texture typically results in a specimen from the conditions by which its substance is formed or engineered, and may be used to quantify its qualities. This project aimed to produce a full process for texture analysis of target samples. It was intended that this process be implemented for CCD or imaging plate diffraction data recorded in the Stanford Synchrotron Radiation Lightsource Beamline 11-3. A process was implemented for smoothly analyzing diffraction images and producing full pole figures. The MATLAB package MTEX formed a base for the implementation of this process. This process was applied to diffraction data from a rolled $\mathrm{Cu}$ specimen. The resulting raw-interpolated and completed pole figures did not sufficiently match to indicate a correct approximation of the orientation distribution function (ODF) for that specimen. A comparative analysis of MTEX and other pole figure inversion algorithms, as applied to this $\mathrm{Cu}$ data, may reveal that another method better approximates the ODF. 


\section{INTRODUCTION}

The texture of a polycrystalline sample is its distribution of crystalline grain orientation in a given direction. Typically, texture results from the conditions of the formation of a sample (either naturally or thorugh an engineered process). Texture of a sample governs some critical material properties of that sample may be deduced. In crystalline samples, most physical properties of interest are anisotropic (have preferred orientations). Additionally, most substances are polycrystalline, making texture analysis frequently necessary [4].

A major motivation for texture analysis is the study of biological structures such as tooth enamel and nacre (see Figure 1). These samples each have significant texture which effects their mechanical properties. The texture of these organically produced structures may be such that it enhances their mechanical properties in directions of importance, such as a chewing direction.

The diffraction of x-rays through a target sample is affected by the texture of that sample. An x-ray source can therefore be used as an illumination source for texture analysis. At the Stanford Synchrotron Radiation Lightsource (SSRL), high intensity x-ray beams provide a suitable source of illumination for x-ray diffraction with goniometers and texture analysis. SSRL beamlines have a setup that would be compatible with a texture analysis system. Experimentation on these beamlines typically involves the illumination of a target sample which may be rotated such that diffraction data can be recorded for various specimen orientations using a CCD or an image plate detector. These area detectors alow quick scanning of the diffraction image. Both current and past SSRL experiments would benefit from a system for texture analysis. A process for texture analysis was thus implemented for this purpose.

\section{DIFFRACTION GEOMETRY}

A variety of notations and formalisms are used to describe diffraction geometry, so a clarification of the geometry in use here is provided. In our case, the target sample is held 
by a goniometer which allows rotation of the sample about a horizontal axis perpendicular to the beam line $(\Delta)$. The diffracted $\mathrm{x}$-rays are visible at the detector as Debye rings corresponding to the intersections of the reciprocal sphere with the Ewald sphere, as shown in Figure 2. These rings each then correspond to diffraction from separate Miller indices. The coordinate system for diffracted points is defined by two coordinates for the location on the Ewald sphere, and a third coordinate for the rotation angle of the sample. These anlges are respectively represented as $\chi, \theta$, and $\Delta$ ) as shown in Figure 3. An equal area projection of the diffraction rings was used, as it is the most suitable for Texture analysis [4], as shown in Figure 6. The resulting planar projections of the Ewald sphere are pole figures. Intensities on pole figures are relative to the density of diffraction points along the intersection of the Ewald and reciprocal spheres. Pole figures are therefore projections of the distribution of crystallite orientations. This distribution of crystallite orientations for a sample is defined as the orientation distribution function (ODF).

\section{METHODS}

The process of analyzing diffraction data to represent it as completed pole figures involves five primary steps, as outlined below [3]:

1. Calibration of the diffraction pattern to account for imaging distortions and extraction of relative intensity peaks from the diffraction image

2. Converting diffraction data into partial and partial-interpolated pole figures

3. Calculation of the ODF from partial pole-figures

4. Plotting of completed pole figures from the ODF

5. Comparison of completed pole figure to the partial pole figure to assess the accuracy of the odf.

3.1 Calibration and the extraction of peaks 
A suitable impelementation of this first step was available, leaving the final four steps to be implemented. This first step can be accomplished using the open-source project Area Diffraction Machine. This program reads a diffraction image, such as in Figure 7, and records peaks along the diffraction rings. These intensity peaks are written to ASCII files with their corresponding $\Delta, \chi, 2 \theta$, and relative intensity values.

\subsection{Converting diffraction data into pole figures}

A process was implemented in MATLAB to convert these peaklist values to Euler angles [5]. The following relations hold for the diffraction geometry described in Section 2.

The spherical law of cosines [2] is applied to the geometry in Figure 3,

$$
\begin{gathered}
\cos (\psi)=\cos \left(\frac{\pi}{2}-\theta\right) \cos (\Delta)+\sin \left(\frac{\pi}{2}-\theta\right) \sin (\Delta) \cos \left(\chi-\frac{\pi}{2}\right) \\
\cos (\psi)=\sin (\theta) \cos (\Delta)+\cos (\theta) \sin (\Delta) \sin (\chi)
\end{gathered}
$$

From Equation 2, the Euler angle $\psi$ can then be calculated. The spherical law of sines [2] is applied to the same geometry,

$$
\begin{aligned}
& \frac{\sin (\pi-\phi)}{\sin \left(\frac{\pi}{2}-\theta\right)}=\frac{\sin \left(\chi-\frac{\pi}{2}\right)}{\sin (\psi)} \\
& \sin (\phi)=-\frac{\cos (\theta) \cos (\chi)}{\sin (\psi)}
\end{aligned}
$$

For the special case when $\Delta=0$, Equations 1 and 2 reduce as expected,

$$
\begin{gathered}
\cos (\psi)=\sin (\theta) \cos (0)+\sin (\theta) \sin (0) \sin (\chi) \\
\cos (\psi)=\sin (\theta) \\
\sin (\phi)=-\frac{\cos (\theta) \cos (\chi)}{\sin \left(\frac{\pi}{2}-\theta\right)}
\end{gathered}
$$




$$
\sin (\phi)=-\cos (\chi)
$$

The angles $\phi$ and $\psi$ are real-valued. Since the range of $\operatorname{asin}$ is limited to $\left[-\frac{\pi}{2}, \frac{\pi}{2}\right], \phi$ is then defined as a piecewise function to ensure full range, with the critical value separating its quadrants defined as $\chi_{0}$,

$$
\cos \left(\chi_{0}\right)=\frac{\sin \left(\operatorname{acos}\left(\frac{\sin (\theta)}{\cos (\Delta)}\right)\right)}{\cos (\theta)}
$$

The relation between $\chi$ and $\chi_{0}$ can then be used ensure that $\phi$ is in the correct quadrant,

$\phi^{\prime}= \begin{cases}\phi & \left.\left(\chi_{0} \leq \chi<\pi-\chi_{0} \text { and } 0 \leq \Delta\right) \text { or }\left(\left(0 \leq \chi<\pi+\chi_{0}\right) \text { or }\left(\chi>2 \pi-\chi_{0}\right)\right) \text { and } \Delta<0\right) \\ \pi-\phi & \left(\left(\left(\pi-\chi_{0} \leq \chi<2 \pi\right) \text { or } \chi<\chi_{0}\right) \text { and } 0 \leq \Delta\right) \text { or }\left(\left(\pi+\chi_{0} \leq \chi<2 \pi-\chi_{0}\right) \text { and } \Delta<0\right)\end{cases}$

An equal-area projection onto a planar surface tangent to the diffraction sphere (as represented in Figure 6) is then applied to the Euler angles, defined by [4]

$$
\sin \left(\psi^{\prime}\right)=\sqrt{2} \sin \left(\frac{\psi}{2}\right)
$$

\subsection{Calculation of the $O D F$}

The calculation of the ODF is a complex mathematical process. To accomplish this, sets of pole figures must be together inverted to construct an approximation of the ODF. This inversion process is analagous to tomography, in that a 3D structure must be reconstructed from its 2D projections. As such, the solutions are not unique, although algorithms for this inversion process may well approximate the ODF with sufficient data.

The threetuples (Intensity, $\phi^{\prime}$, and $\psi^{\prime}$ ) resulting from the end of Section 3.2 are binned into user-defined polar area segments (a typical size being $1^{\circ} \times 1^{\circ}$ ) with similar-binned points being averaged together. The binned data is written as a raw pole 
figure file in Philips diffractometer format. This file can then be read in as needed to display a pole figure, or combined with other pole figures and further processed to produce an ODF. Each pole figure corresponds to a separate Miller index. These pole figures are 'raw' and incomplete due to the limitations of data coverage. Since the range of sample rotation is limited to approximately $\left[-50^{\circ}, 50^{\circ}\right]$ in our case, some regions of these pole figures cannot be scanned. The resulting data coverage fills an area as shown in Figure 8.

The raw pole figure data is read into MATLAB using functionality provided by the software package MTEX [1] (a set of MATLAB functions). The MTEX algorithm is a recently developed algorithm that was implemented in a set of open-source MATLAB functions. The general structure of this algorithm begins with the selection of a radial basis function on $\mathrm{SO}(3) \rightarrow \mathbb{R}$. An equidribution of crystal orientations is then determined. The unknown ODF is then approximated by a finite linear combination of radially symmetric unimodal ODFs. The coefficients of the linear combination and normalization coefficients are then determined as a minimization problem by a mean square like function with the additional constraints that the resulting ODF is non-negative and smooth. Additionally, this method allows for an arbitrary crystal and diffraction geometry as well. For a more detailed description of the MTEX algorithm, see the paper by Hielscher and Schaeben [1]. An estimated ODF is then calculated using the MTEX pole-figure inversion algorithm.

\subsection{Plotting pole figures}

The 'completed' pole figures can then be calculated and plotted from the ODF. The incomplete-interpolated pole figures are also plotted from the raw pole figure files for comparison. Plotting these pole figures is simply accomplished through the use of MTEX's functions.

\subsection{Comparison of the pole figures as a check of accuracy}

Comparison of completed pole figure to the partial pole figure to assess the accuracy of the odf. An iterative path may be taken to produce a series of better 'complete' pole figures based on a comparison between the interpolated and incomplete pole figures, either 
programmatically or by user query. If, either computationally or by user inspection, the completed and incomplete-interpolated pole figures do not sufficiently correlate, the ODF may be recalculated with higher resolution for a better approximation.

\subsection{Experimental evaluation of this process}

In order to evaluate the correctness of this process and the MTEX algorithm, diffraction data was taken for $\Delta$ values between $-50^{\circ}$ and $50^{\circ}$ in increments of $1^{\circ}$ for a well textured (assumedly rolled) $\mathrm{Cu}$ specimen. This data was taken on SSRL Beam Line 11-3 (shown in Figures 4 and 5) using a Mar345 imaging plate detector. For specifications of the experimental setup, see Table. The Cu diffraction data was plotted in pole figures (see Figures 9-12) and interpolated over constant $\psi$ values (see Figures 13-16). The MTEX pole

figure inversion method was applied to the raw pole figures, resulting in the ODF shown in Figure 21. From the computed ODF, completed pole figures (see Figures 17-20) were plotted and compared with the raw pole figures as a check.

\section{RESULTS}

In comparing the interpolated raw and completed pole figures, the $\{111\}$ (Figures 13 and 17) and \{220\} (Figures 15 and 19) pole figures exhibited moderate similarity. The $\{200\}$ (Figures 14 and 18) and \{311\} (Figures 16 and 20) pole figures showed little similarity. The completed pole figures only slightly resemble their corresponding raw pole figures, indicating that the MTEX algorithm did not produce a sufficiently accurate ODF for the $\mathrm{Cu}$ specimen.

\section{CONCLUSIONS}

From the apparent inconsistencies between the raw-interpolated and completed pole figures, it is clear that the MTEX algortihm did not produce a sufficient approximation of the specimen ODF. In future work, the MTEX algorithm may be replaced with the older WIMV algorithm, which is known to be robust in obtaining the ODF from incomplete pole figure data. A comparative analysis of the results from these and other algorithms would provide a better benchmark for the selection of a pole figure inversion method. 
Additionally, a complete automation of this procedure would be desired.

\section{ACKNOWLEDGEMENTS}

I much appreciate the continued guidance and support from my mentor, Apurva Mehta. Additional thanks go to Matthew Bibee, James (Randy) Groves, Marc Davidson, and Karen Magid. I also thank the DOE, SULI Program, the Stanford Linear Accelerator Center, and the Stanford Synchrotron Radiation Lightsource laboratory for cooperating to provide myself and others with engaging undergraduate research opportunities.

\section{REFERENCES}

[1] Journal of Applied Crystallography, Vol. 41, No. 6. (December 2008), pp. 1024-1037.

[2] Weisstein, Eric W. S̈pherical Trigonometry.ت̈rom MathWorld-A Wolfram Web Resource. http://mathworld.wolfram.com/SphericalTrigonometry.html

[3] H.-R. Wenk and S. Grigull (2003). Synchrotron texture analysis with area detectors. J. Appl. Cryst. 36, 1040-1049.

[4] U.F. Kocks and J.S. Kallend. In: U.F. Kocks, C.N. Tom and H.-R. Wenk, Editors, Texture and Anisotropy, Cambridge University Press, Cambridge, UK (1998), p. 54, 104-124.

[5] Weisstein, Eric W. "Euler Angles." From MathWorld-A Wolfram Web Resource. http://mathworld.wolfram.com/EulerAngles.html 
Figures:

\begin{tabular}{|c|c|c|c|}
\hline & Harmonic & MTEX & WIMV/APC \\
\hline \hline Flexibility of Implementation & $\times[4]$ & $\checkmark[1]$ & $\checkmark[4]$ \\
\hline Ghost Correction & $\times[4]$ & & $\checkmark[4]$ \\
\hline Incomplete Pole Figures & $\times[4]$ & & $\checkmark[4]$ \\
\hline Low Symmetry Samples & $\times[4]$ & & $\checkmark[4]$ \\
\hline Complexity of Implementation & $\times[4]$ & $\checkmark[1]$ & \\
\hline Economy of Implementation & $\checkmark[4]$ & $\checkmark[1]$ & $\times[4]$ \\
\hline Poor Quality Diffraction Data & $\checkmark[4]$ & & $\times[4]$ \\
\hline Susceptibility to Noise & $\checkmark[4]$ & & $\times[4]$ \\
\hline Area Detectors & & $\checkmark[1]$ & \\
\hline Sharp Textures & & $\checkmark[1]$ & \\
\hline High Resolution Pole Figures & & $\checkmark[1]$ & \\
\hline
\end{tabular}

istics of pole figure inversion algorithms. Checks indicate a favorable characteristic, and crosses indicate unfavorable characteristics. The adjacent bracketed numbers are the related references.

\begin{tabular}{|c|c|}
\hline & Specifications for $\mathrm{Cu}$ diffraction experiment \\
\hline \hline Beam Energy & $12.7 \mathrm{keV}$ fixed \\
\hline Beam Wavelength & $.97 \AA$ \\
\hline Spot Size & $150 \times 150 \mu \mathrm{m}$ \\
\hline$\Delta$ Range & $-50^{\circ}$ to $50^{\circ}$ \\
\hline$\Delta$ Step Size & 1 \\
\hline Sample Type & Thin $\mathrm{Cu}$ foil \\
\hline
\end{tabular}

Table 2: Specifications

for the evaluational diffraction experiment. 


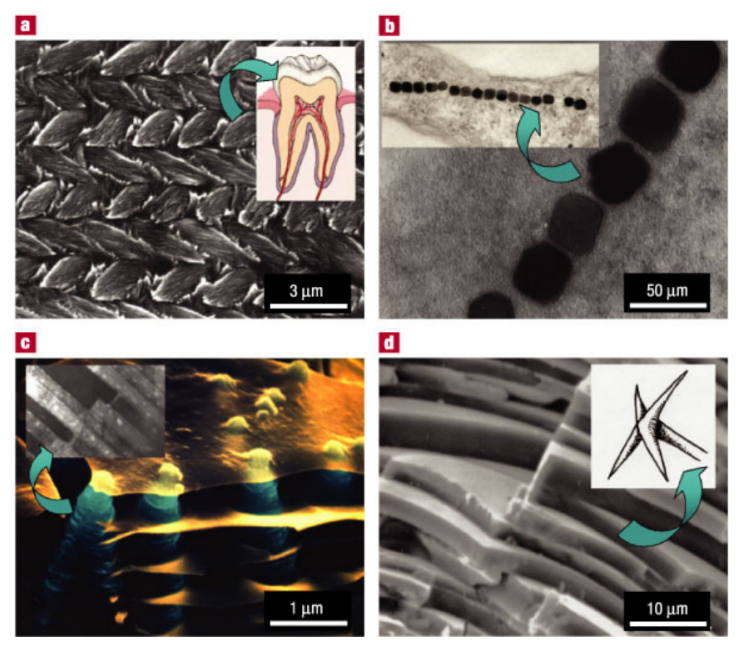

Figure 1: Mouse enamel, magnetotactic bacterium, abalone aragonite platelets, and sponge spicule each have texture.

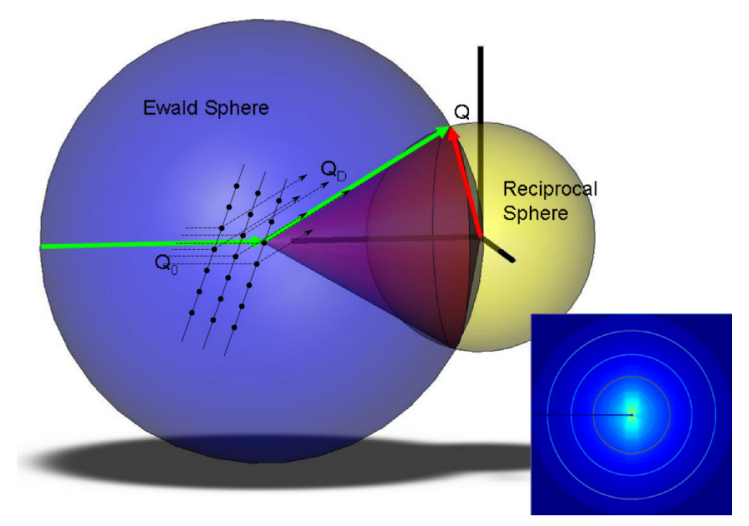

Figure 2: The ewald and reciprocal spheres. The intersection of the surfaces of these spheres is a ring. Diffraction data therefore appears as a series of concentric rings on the detector plane. 


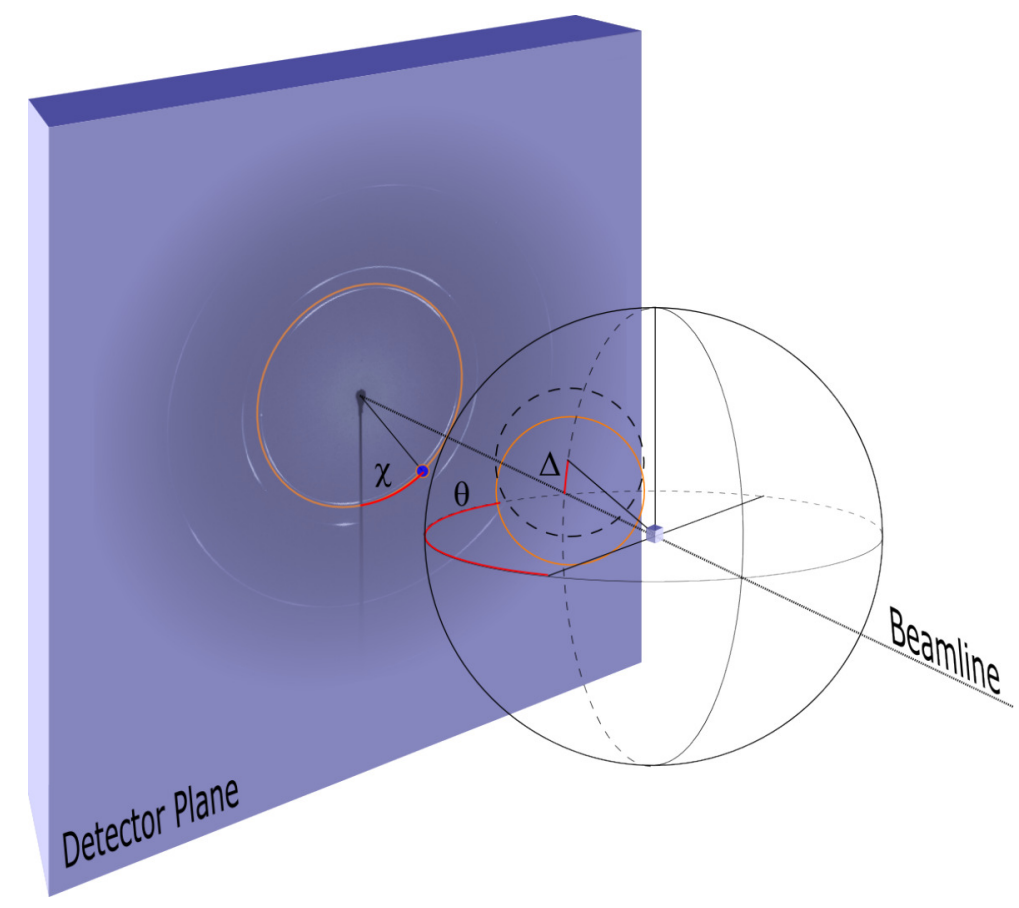

Figure 3: The diffraction geometry coordinate system used for the peak list data sets. The sample is rotated along the $\Delta$ angle axis.

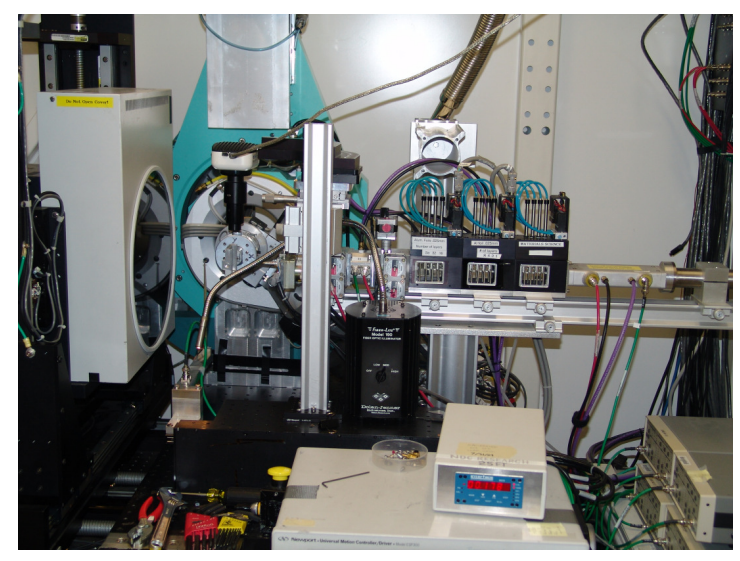

Figure 4: A view of Beamline 11-3 from the hutch door. The x-ray beam comes from right to left. 


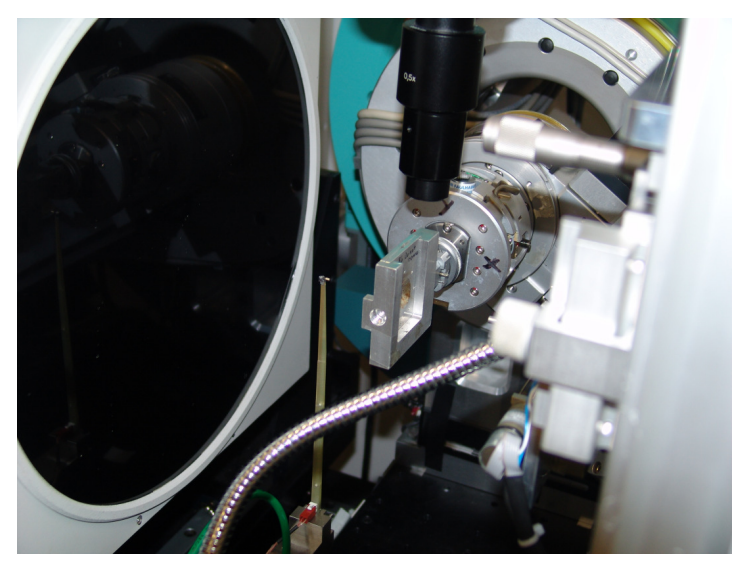

Figure 5: A view of the $\mathrm{Cu}$ sample and specimen holder. The goniometer arm rotates the sample in place. 


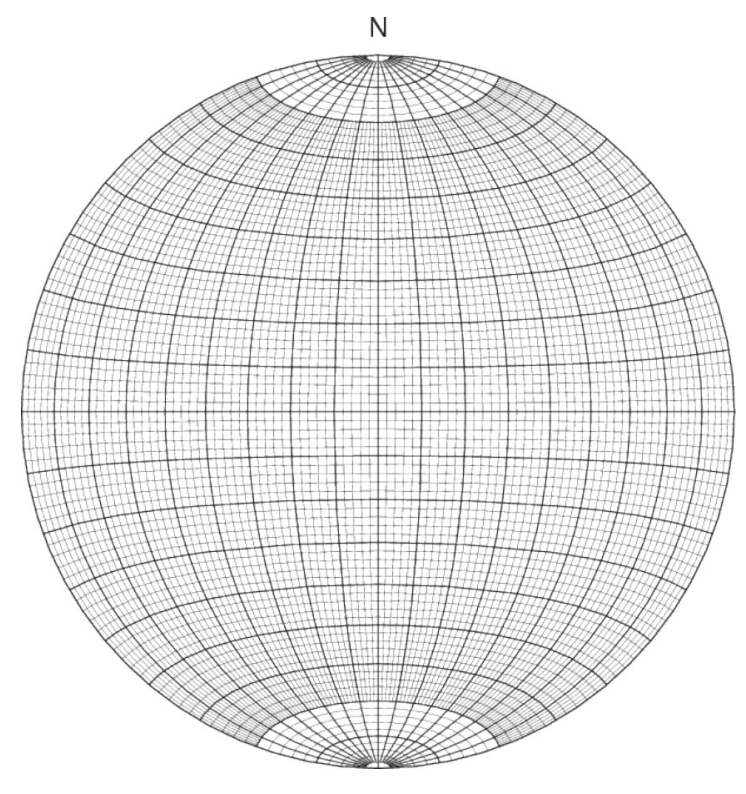

Figure 6: An equal area net on a sphere. A flattened hemisphere of the equal area net is the equal area projection on a planar surface. 


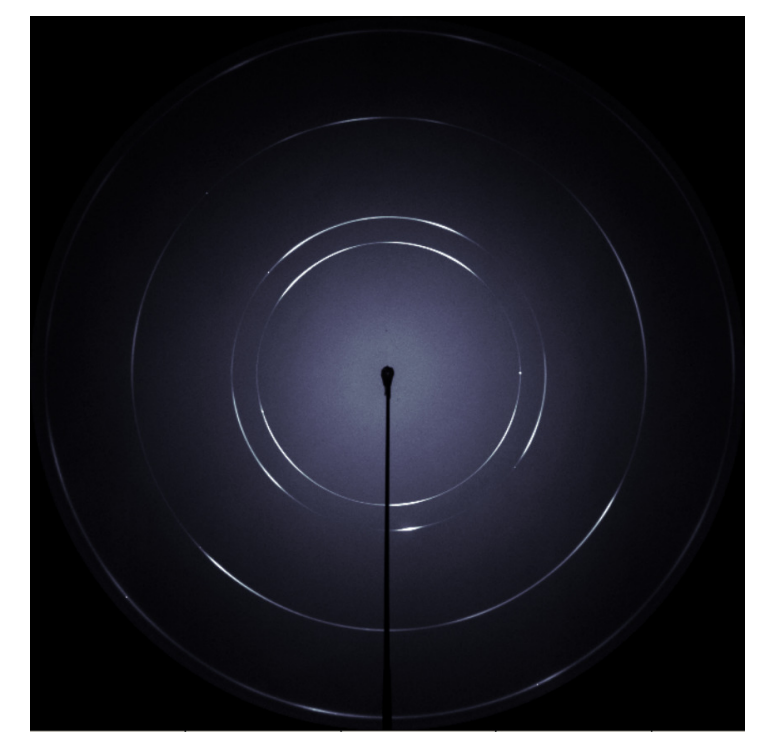

Figure 7: An example of a diffraction image recorded from a Mar345 imaging plate detector, displayed in Area Diffraction Machine.

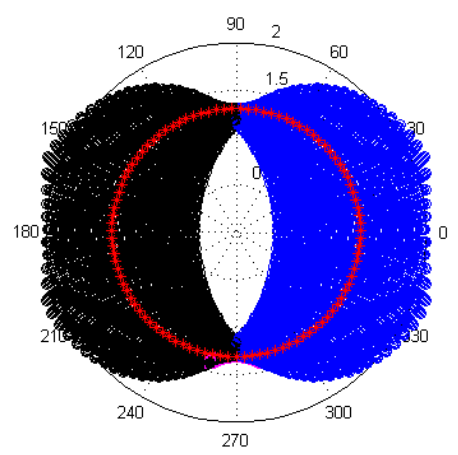

Figure 8: An example of the range of coverage of diffraction data for this experimental setup. 


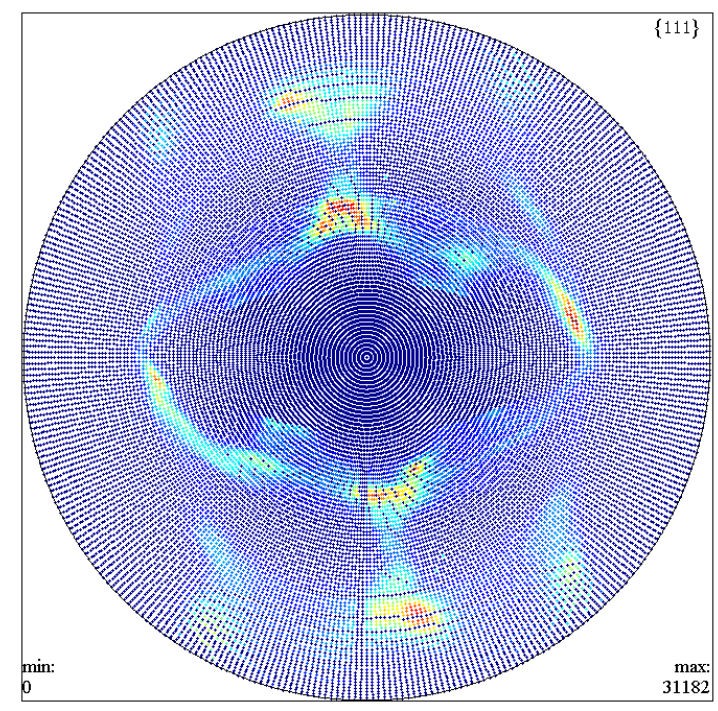

Figure 9: The (111) pole figure for the $\mathrm{Cu}$ diffraction data set.

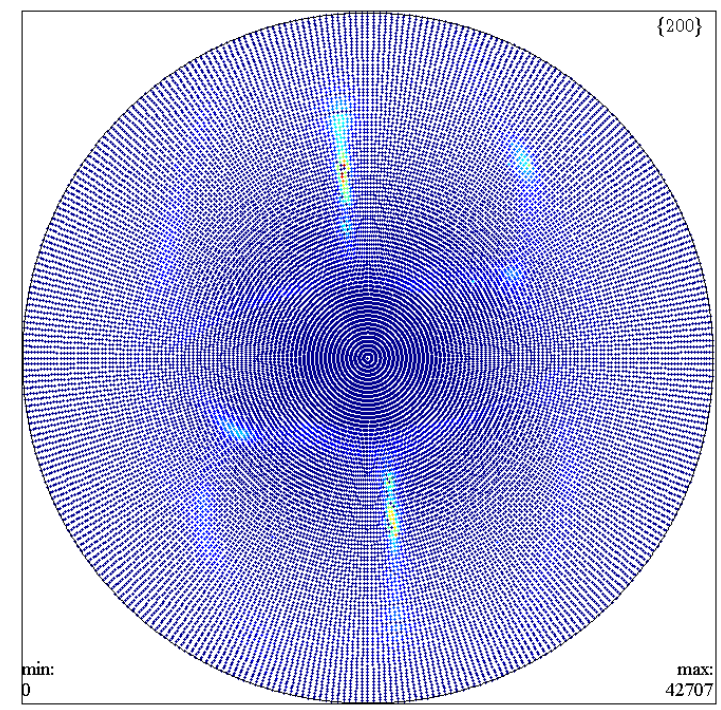

Figure 10: The (200) pole figure for the $\mathrm{Cu}$ diffraction data set. 


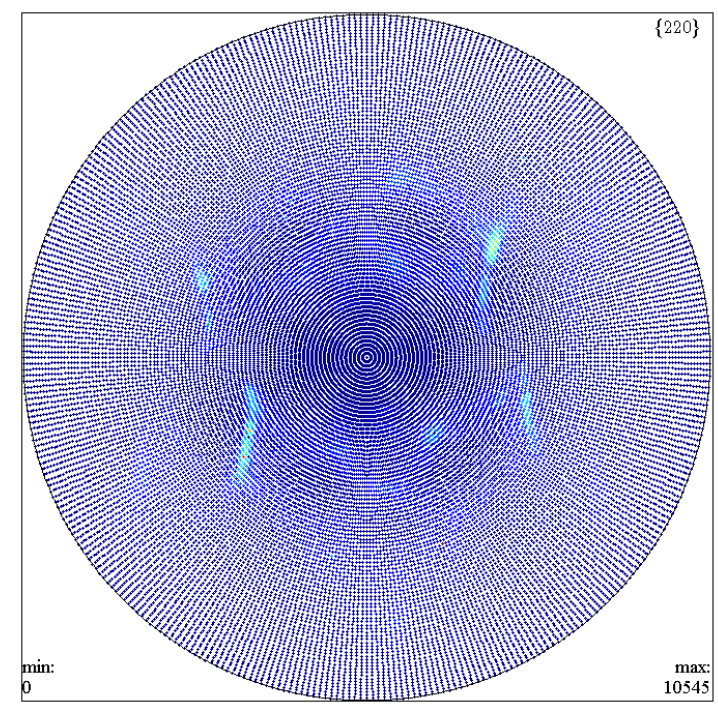

Figure 11: The (220) pole figure for the $\mathrm{Cu}$ diffraction data set.

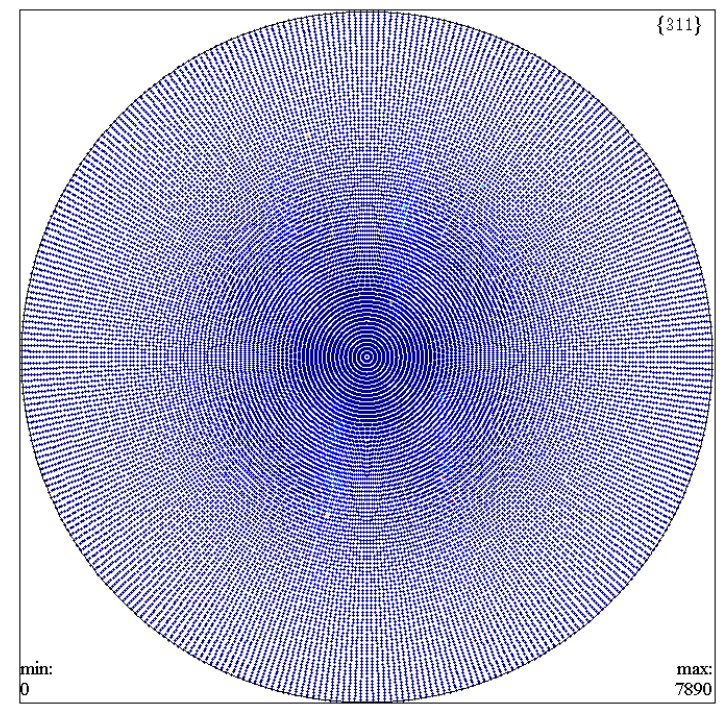

Figure 12: The (311) pole figure for the $\mathrm{Cu}$ diffraction data set. 


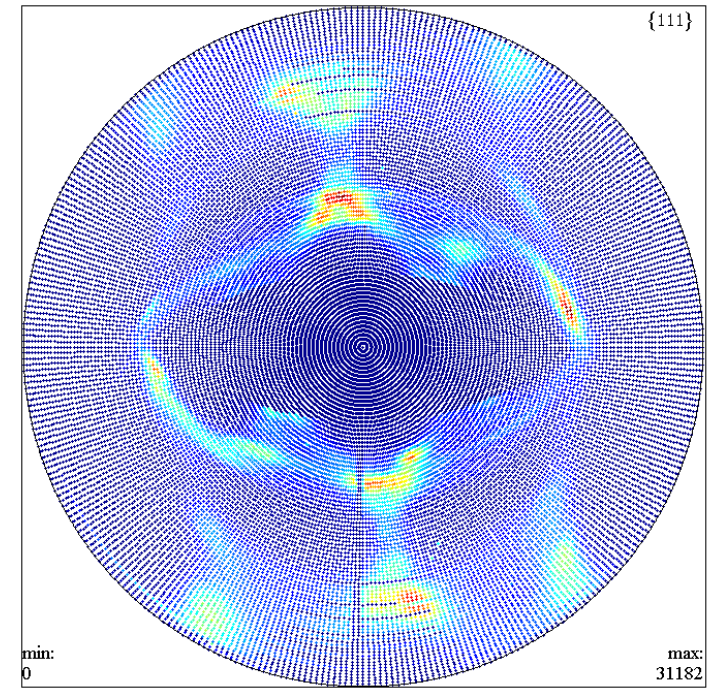

Figure 13: The (111) interpolated pole figure for the $\mathrm{Cu}$ diffraction data set.

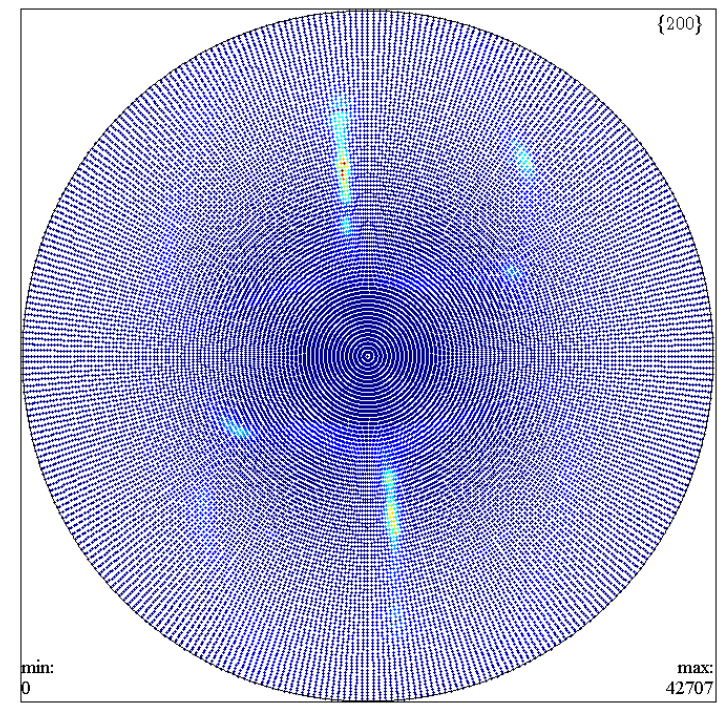

Figure 14: The (200) interpolated pole figure for the $\mathrm{Cu}$ diffraction data set. 


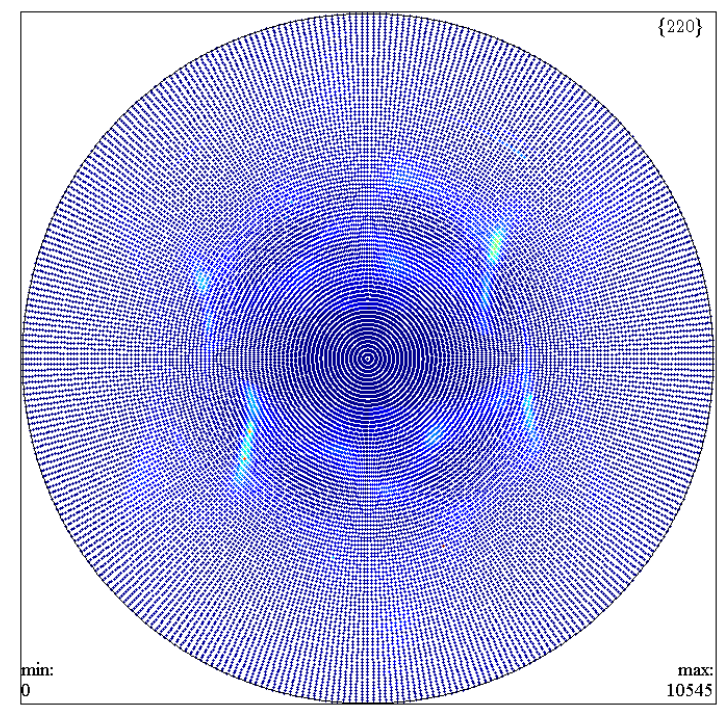

Figure 15: The (220) interpolated pole figure for the $\mathrm{Cu}$ diffraction data set.

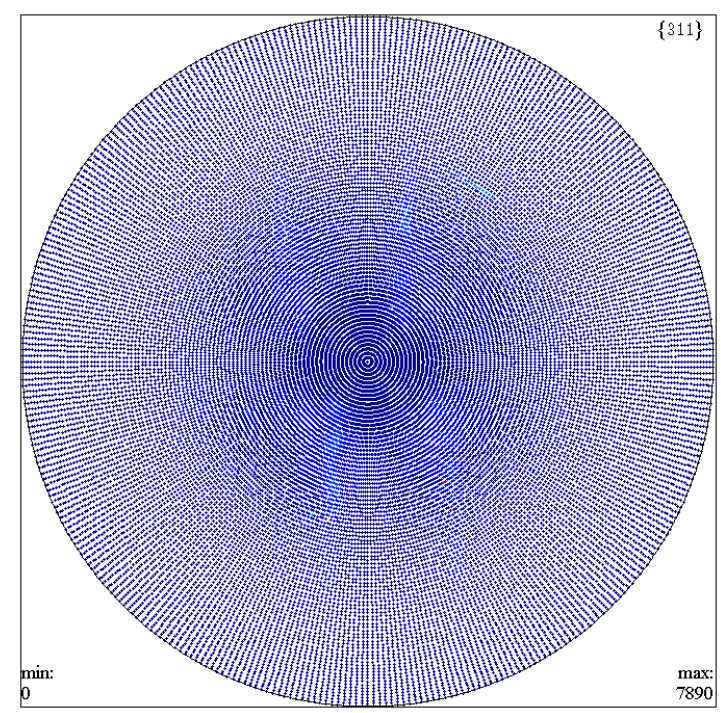

Figure 16: The (311) interpolated pole figure for the $\mathrm{Cu}$ diffraction data set. 


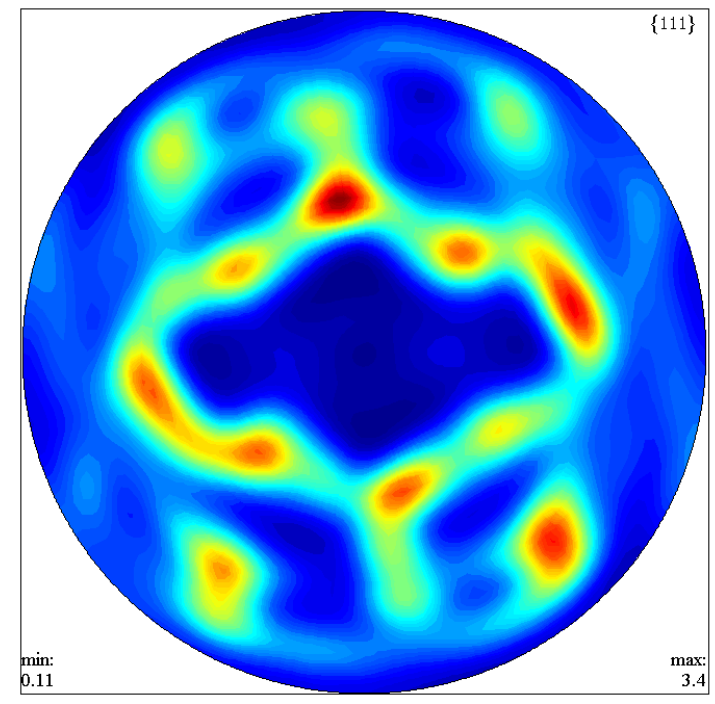

Figure 17: The (111) completed pole figure for the $\mathrm{Cu}$ diffraction data set. 


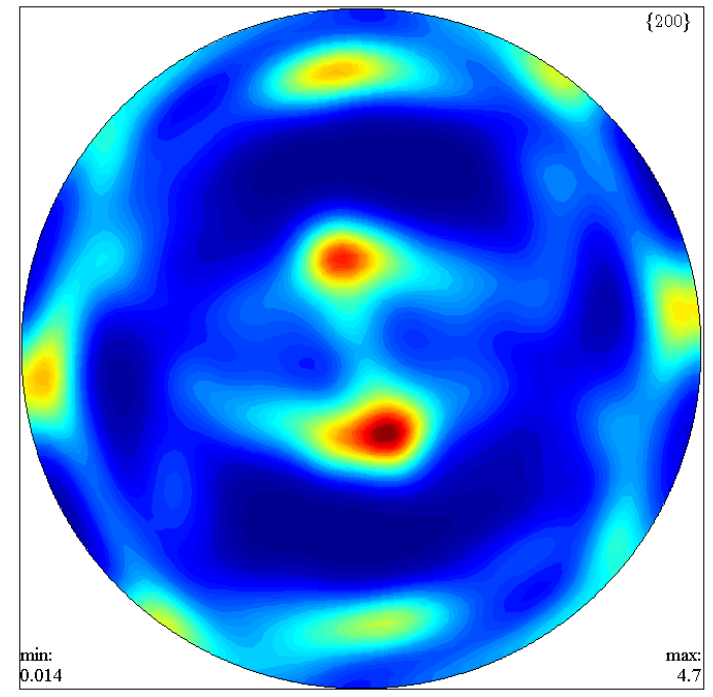

Figure 18: The (200) completed pole figure for the $\mathrm{Cu}$ diffraction data set.

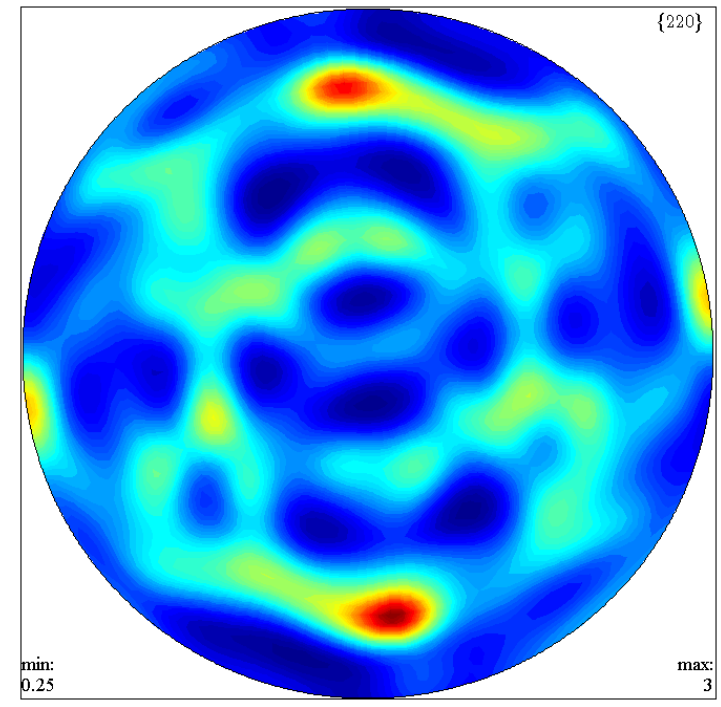

Figure 19: The (220) completed pole figure for the $\mathrm{Cu}$ diffraction data set. 


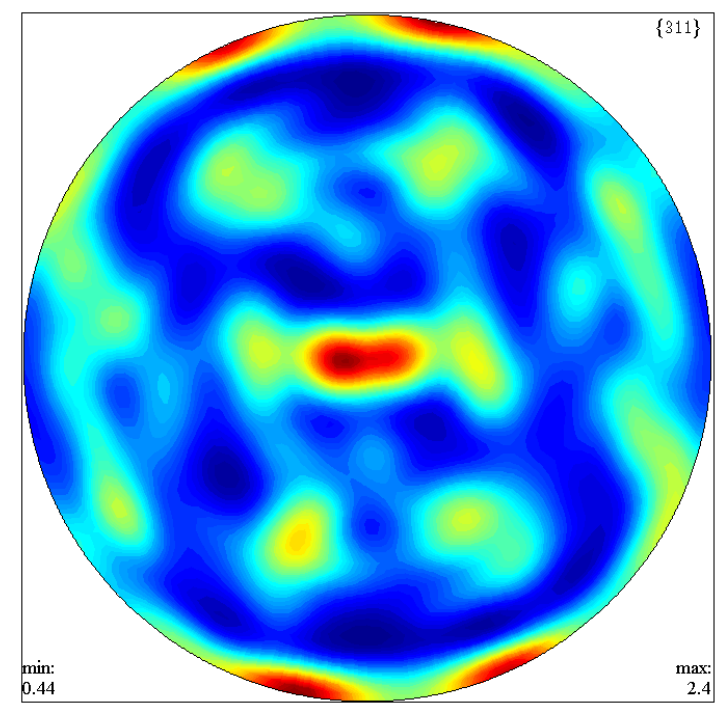

Figure 20: The (311) completed pole figure for the $\mathrm{Cu}$ diffraction data set.

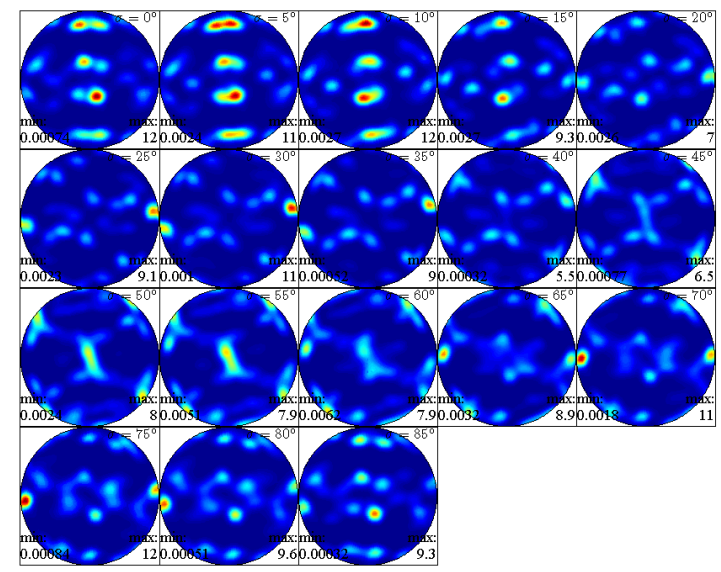

Figure 21: The ODF for the $\mathrm{Cu}$ diffraction data set. 